\title{
Étude de la diversité génétique du blé tendre. II. Application à la prédiction de l'hétérosis
}

\author{
B Picard *, G Branlard, FX Oury, P Bérard, M Rousset \\ INRA, station d'amélioration des plantes, domaine de Crouelle, 63039 Clermont-Ferrand Cedex, France
}

(Reçu le 17 janvier 1992: accepté le 5 juin 1992)

\begin{abstract}
Résumé - L'éloignement génétique entre 33 lignées de blé tendre ayant servi à la production de 34 hybrides, a été estimé à partir de données biochimiques et à partir de l'étude des généalogies. Un dispositif multilocal a permis d'évaluer les hybrides et leurs parents, et de calculer les hétérosis pour le rendement, par rapport aux parents moyen et supérieur. II n'apparaît aucune corrélation entre distances biochimiques et hétérosis, pas plus qu'entre le coefficient de parenté et l'hétérosis. L'intérêt de différentes mesures de distance pour la prédiction de l'hétérosis est discuté.
\end{abstract}

blé hybride / diversité génétique / distance / hétérosis

Summary - A study of genetic diversity in wheat. II. Application to the prediction of heterosis. Genetic divergence between 33 wheat lines used to produce 34 hybrids was estimated from biochemical data and a study of pedigrees. A multilocation experiment permitted evaluation of parents and hybrids, and the calculation of mid-parent and high-parent heterosis (table I). There was no correlation, either between biochemical distances and heterosis, or between coefficients of parentage according to Malecot and heterosis (fig 1, tables II and III). The use of different distances for heterosis prediction has been discussed.

hybrid wheat / genetic diversity / distance / heterosis

\section{INTRODUCTION}

Les travaux de East et Shull (East, 1936) sur le mais ont montré que le produit du croisement entre 2 lignées homozygotes (lignées pures), était généralement plus vigoureux que ses parents (manifestation d'une vigueur hybride, ou hétérosis). Pour le blé, les premières études de I'hétérosis ont débuté en 1919 avec les travaux de Freeman, qui notait que l'intensité du phénomène était fonction du croisement effectué.

Les 2 principales explications du phénomène d'hétérosis sont celles faisant appel à la dominance ou à la superdominance. L'hétérosis pour un caractère donné est alors d'autant plus élevé que les parents de l'hybride sont complémentaires pour les locus impliqués dans la variation de ce caractère. Cette complémentarité est liée à l'éloignement génétique entre les parents des hybrides, qui peut être estimé par le calcul de distances (Lefort-Buson, 1985a,b; Brunel, 1985).

De fait, chez le maïs, de nombreux travaux (Frei et al, 1986; Lee et al, 1989; Smith et Smith, 1989b; Godshalk et al, 1990; Melchinger et al, 1990a,b; Smith et al, 1990) ont été accomplis ces dernières années pour étudier la relation entre I'hétérosis et différentes distances basées soit sur les généalogies, soit sur la variabilité de caractères agromorphologiques, soit sur le polymorphisme biochimique ou moléculaire.

Cependant, chez une plante autogame comme le blé, les travaux de ce type ont jusqu'à présent été très limités, en raison des difficultés de production des semences $F_{1}$. En effet, la voie manuelle (castrations suivies de pollinisations croisées) n'était pas praticable à grande échelle, et

\footnotetext{
- Adresse actuelle : INRA, laboratoire croissance et métabolisme des herbivores, UR croissance musculaire, Theix, 63122 SaintGenès-Champanelie, France
} 
la voie génétique (Kihara, 1951; Fusakawa, 1955) s'est avérée très délicate à utiliser, car nécessitant la production de plusieurs types de lignées (lignées mâles-stériles, lignées restauratrices). La liaison entre hétérosis et distance interparentale ne pouvait alors être étudiée qu'au niveau de dispositifs expérimentaux restreints, avec une évaluation des génotypes sur une seule ligne (Shamsuddin, 1985; Xu et Wang, 1982), ou au travers de l'hétérosis résiduel existant en $F_{2}$ (Cox et Murphy, 1990).

L'apparition des agents chimiques d'hybridation (produits qui induisent une stérilité mâle par pulvérisation sur les plantes à un stade phénologique approprié) a permis de lever les contraintes pesant sur la production des semences hybrides. Pour cette étude, la possibilité de produire des $F_{1}$ par voie chimique nous a permis de disposer d'un grand nombre d'hybrides, avec des quantités de semences suffisantes pour pouvoir réaliser des expérimentations multilocales en grandes parcelles, c'est-à-dire dans des conditions proches de celles de la pratique agricole courante. Nous avons pu ainsi étudier la relation entre différentes distances interparentales calculées à partir de données biochimiques et à partir de l'étude des généalogies, et l'hétérosis rendement obtenu chez les hybrides correspondants.

Ce travail a été réalisé dans le cadre d'une collaboration entre I'INRA et le *GIE «Hybriblé».

\section{MATÉRIEL ET MÉTHODES}

L'ensemble du matériel végétal étudiè a été fourni par les différents membres du GIE “Hybriblé" et par les partenaires INRA de ce GIE, qu'il s'agisse des lignées parentales ou des hybrides. Ces derniers ont été produits avec un des gamétocides expérimentaux d'Orsan.

L'expérimentation a porté sur 48 hybrides obtenus par voie chimique à partir de 47 lignées parentales, sans plan de croisement défini. Ces 48 hybrides ont été évalués en même temps que leurs parents en 1989, en 3 lieux (Clermont-Ferrand, Le Moulon et Mons-en-Chaussée). Le même dispositif expérimental comprenant 3 blocs complets randominés a été utilisé dans chacun des lieux, la surface parcellaire étant au minimum de $3,5 \mathrm{~m}^{2}$.

Le produit gamétocide utilisé étant encore en phase d'expérimentation au moment de l'étude, un contrôle de la pureté des hybrides a été nécessaire. Pour un certain nombre de $F_{1}$, ce contrôle a été réalisé par observation des caractères d'aristation et de glaucescence (ceux-ci, ayant une hérédité simple, permettent dans les cas favorables de distinguer les hybrides purs des autofécondations et des hybrides hors-type). Les $F_{1}$ pour lesquelles cette observation ne permettait pas de conclure ont été analysées par électrophorèse des gluténines de haut poids moléculaire en milieu Sodium Dodécyl Sulphate (SDSPAGE). La méthode de Laemmli (1970), modifiée par Payne et al (1979), a été adaptée dans ce but sur cuve "Electronucleonics", ce qui permettait la migration simultanée de 20 plaques de 24 dépôts chacune. Pour chaque combinaison hybride, 48 grains ont été analysés.

Les différentes distances interparentales qui ont été mises en œuvre sont décrites en détail dans Picard et al (1992). II s'agissait du coefficient de parenté de Malécot (1948), et de distances biochimiques calculées à partir des diagrammes obtenus par électrophorèse des protéines de réserve du grain.

Ces distances biochimiques correspondaient:

- à des distances estimées à partir de la présenceabsence des différents allèles des gluténines de hauts poids moléculaires;

- à des distances basées sur la présence-absence des bandes gliadines et l'intensité relative de ces bandes.

Pour les gluténines et les gliadines, il y a eu à chaque fois 2 calculs : I'un utilisant la formule de la distance absolue et l'autre la formule de la distance du $\chi^{2}$ (cette dernière confère un poids relatif plus important aux individus présentant un diagramme rare).

Les différents traitements statistiques et graphiques ont ètè effectués à l'aide du logiciel "S", sous système d'exploitation “UNIX". Pour le calcul des distances, et en particulier le coefficient de parenté, plusieurs programmes ont été écrits en Fortran.

\section{RÉSULTATS}

\section{Pureté des hybrides}

Nous n'avons retenu que les combinaisons pour lesquelles le taux de plantes de type femelle ou hors-type était inférieur à 30\% (seuil en-dessous duquel le jugement des performances des hybrides n'est pas influencé par leur degré d'impureté). Après ce tri, il restait 34 hybrides et les 33 parents correspondants.

\footnotetext{
"Le GIE «Hybriblé» regroupe les établissements de sélection Blondeau, Momont, Orsem et UCASP, et la firme Orsan (pour la recherche sur les gamétocides).
} 


\section{Rendements des hybrides}

Le tableau I donne quelques caractéristiques des essais réalisés dans les 3 lieux. Ces essais apparaissent de bonne qualité (niveau des rendements élevé et valeurs des coefficients de variation indiquant une bonne précision). En chacun des lieux le rendement moyen des 34 hybrides est équivalent au rendement moyen des 4 témoins (Camp-Rémy, Festival, Promentin et Thésée), mais les meilleurs hybrides sont partout supérieurs aux meilleures lignées : à Clermont, le meilleur hybride réalise un rendement de 99,4 q/ ha contre $93 \mathrm{q} / \mathrm{h}$ a pour Promentin, lignée la plus productive; au Moulon, le rendement de la meilleure $F_{1}$ est de 116,1 q/ha, contre 109,2 q/ha pour la meilleure lignée; à Mons, les valeurs sont de $103,3 \mathrm{q} /$ ha pour l'hybride le plus productif et de 97,8 q/ha pour la meilleure lignée, Thésée. Globalement les valeurs moyennes d'hétérosis sont assez importantes et équivalentes pour les différents lieux (tableau I). Pour une étude plus précise de cette expérimentation multilocale, et en particulier une discussion sur l'hétérosis chez le blé, voir Oury et al (1990).

Tableau I. Quelques caractéristiques des essais conduits dans les 3 lieux. Hétérosis PM = hétérosis en pourcentage du parent moyen; hétérosis PS = hétérosis en pourcentage du parent supérieur.

\section{Clermont Le Mons Moulon}

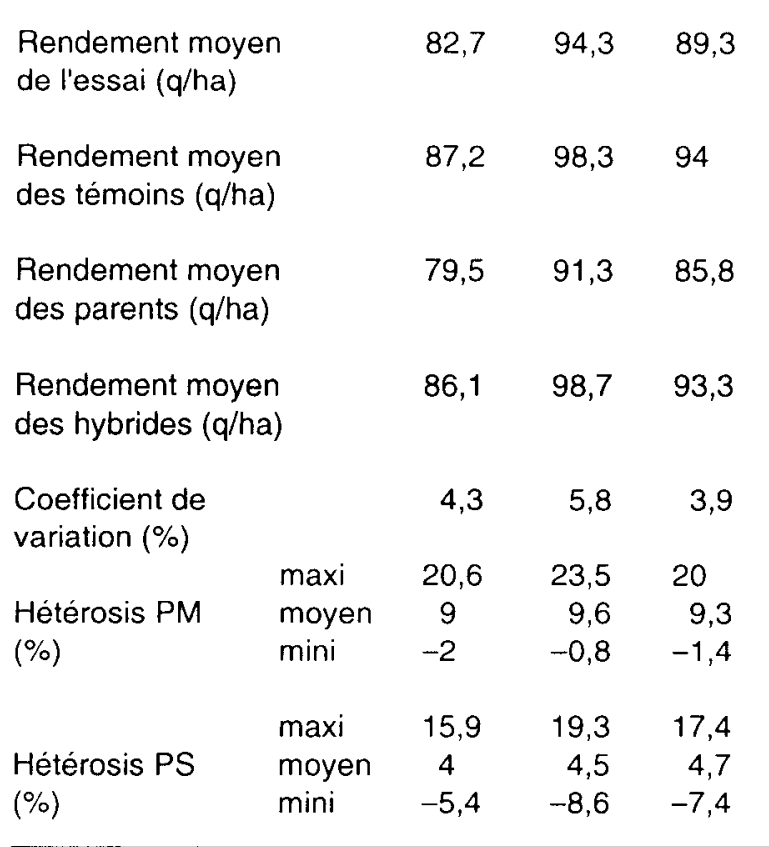

\section{Relation distances - hétérosis}

Les relations entre les différentes distances et l'hétérosis par rapport au parent supérieur sont représentées dans le tableau II et la figure 1. Celles entre distances et hétérosis par rapport au parent moyen figurent dans le tableau II (les représentations graphiques étant très semblables à celles de la figure 1, ne sont pas présentées ici).

II n'apparaît aucune liaison entre les différentes distances et l'hétérosis : aucun coefficient de corrélation n'est significatif au seuil de $5 \%$. Celui obtenu à Mons entre I'hétérosis par rapport au parent moyen et le coefficient de parenté est à la limite de la signification, mais la relation dans ce cas, est due uniquement à un point : si on enlève le point correspondant à ce coefficient de parenté élevé, le coefficient de corrélation prend la valeur $-0,075$, non significative.

\section{DISCUSSION}

\section{Relations entre distances biochimiques et hétérosis}

Si la distance "gluténines" correspond bien à une distance génétique, par contre la distance

Tableau II. Valeurs des coefficients de corrélation linéaire entre I'hétérosis par rapport au parent moyen et les différentes distances pour les 3 lieux (entre parenthèses, même chose mais pour l'hétérosis par rapport au parent supérieur). ${ }^{*}=$ significatif au seuil de $5 \%$ (la valeur critique, pour 33 ddl, est de 0,339 ).

Clermont Le Moulon Mons

$\begin{array}{lccc}\begin{array}{lccc}\text { Distance absolue } \\ \text { allèles gluténines }\end{array} & \begin{array}{c}-0,217 \\ (-0,236)\end{array} & \begin{array}{c}-0,071 \\ (-0,138)\end{array} & \begin{array}{c}0,223 \\ (-0,230)\end{array} \\ \begin{array}{lccc}\text { Distance du chi } 2 \\ \text { allèles gluténines }\end{array} & -0,024 & -0,240 & 0,147 \\ & (-0,128) & (-0,278) & (0,280) \\ \text { Distance absolue } & 0,149 & -0,028 & 0,181 \\ \text { bandes gliadines } & (0,198) & (-0,154) & (0,173) \\ \text { Distance du chi } & 0,196 & -0,166 & 0,179 \\ \text { bandes gliadines } & (0,211) & (-0,248) & (0,089) \\ & & & -0,342 \\ \text { Coefficient de } & -0,013 & -0,163 & -0,056) \\ \text { parenté } & (-0,063) & (-0,056) & (-0,277)\end{array}$



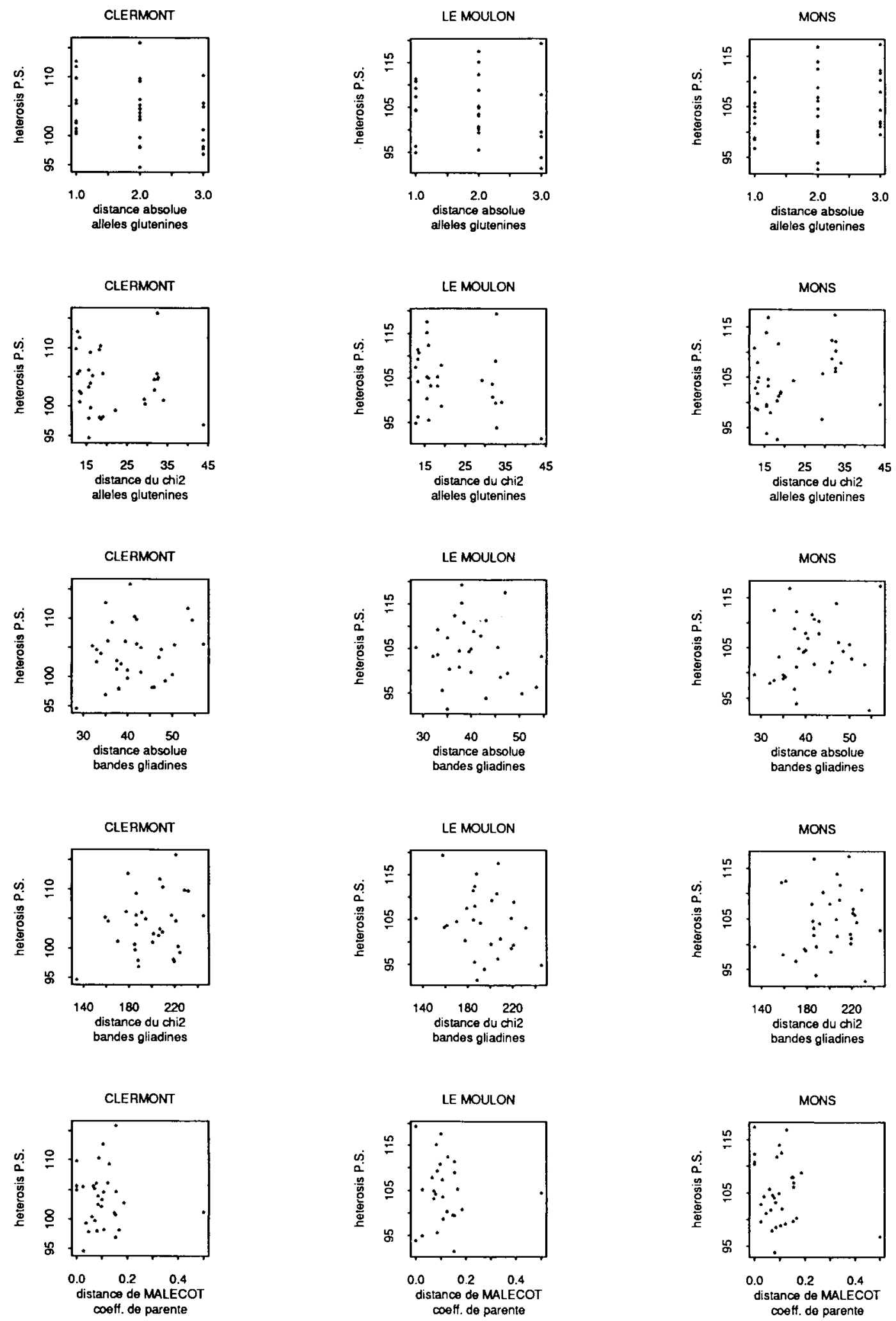

Fig 1. Relations entre les différentes distances et l'hétérosis par rapport au parent supérieur dans les 3 lieux. 
"gliadines" n'en est pas strictement une, puisqu'elle est basée sur la présence-absence de bandes dont le déterminisme génétique n'est pas toujours précisément connu. Un progrès peut donc être attendu d'une lecture génétique des diagrammes gliadines (Sozinov et Poperelya, 1980), bien que ce travail soit très lourd pour une famille multiallélique comme les gliadines (Mecham et al, 1978; Branlard, 1983; Payne et al, 1984) et n'ait de ce fait pas encore abouti.

Quoi qu'il en soit, plusieurs résultats bibliographiques indiquent, comme dans notre étude, une absence de liaison entre distances biochimiques (obtenues le plus souvent par électrophorèse de systèmes enzymatiques) et hétérosis, aussi bien chez le maïs (Frei et al, 1986), que chez le pin (Strauss, 1986) ou la drosophile (Ehiobu et al, 1990). Chez le maîs, Smith et Smith (1989b) ont mis en évidence une liaison significative entre I'hétérosis et une distance biochimique, mais la part de variance expliquée était trop faible pour que la relation soit vraiment utilisable. HeidrichSobrinho et Cordeiro (1975), en étudiant l'aptitude à la combinaison, ont, quant à eux, mis en évidence une liaison nette entre l'AGC et une distance biochimique, mais pas entre I'ASC et cette même distance biochimique. Une liaison positive entre distance biochimique et hétérosis peut également apparaître lorsque les hybridations ont lieu entre du matériel apparenté (Frei et al, 1986; Strauss, 1986), mais cette liaison risque d'être alors de peu d'intérêt car souvent les combinaisons les plus intéressantes sont celles qui font intervenir des parents peu ou pas apparentés.

L'absence de relation indique que les locus impliqués dans les distances biochimiques, soit ne contribuent pas à l'hétérosis, soit ne sont pas liés aux locus impliqués dans l'hétérosis. Ceci n'est pas surprenant compte tenu du faible nombre de locus que les distances biochimiques mettent en jeu : il devient alors très improbable que ces locus puissent représenter correctement l'hétérozygotie aux locus impliqués dans l'hétérosis.

\section{Relations entre coefficient de parenté et hétérosis}

Les valeurs d'apparentement ne sont pas régulièrement réparties dans l'ensemble des 34 hybrides étudiés. En effet, il n'existe pas d'hybrides dont les parents ont un coefficient de parenté compris entre 0,2 et 0,5 (fig 1). Cette faible variabilité d'apparentement au sein de la population étudiée peut être une de raisons à l'origine de l'absence de liaison entre hétérosis et apparentement.

Les résultats concernant la relation entre coefficient de parenté et hétérosis sont variables selon les espèces.

Chez le maïs, il apparaît une liaison assez forte entre hétérosis et distance généalogique (Paterniani et Lonnquist, 1963; Moll et al, 1962 et 1965; Smith et Smith, 1989b; Smith et al, 1990). Cependant, ce résultat ne présente qu'un intérêt relatif, car s'il permet de définir quels sont les croisements à ne pas faire (ceux entre lignées apparentées), il ne permet pas de choisir les croisements à réaliser parmi tous ceux qui sont possibles entre des lignées non apparentées.

Chez les autogames, les résultats vont dans le sens de ceux que nous avons obtenus, la relation entre coefficient de parenté et hétérosis apparaît trop faible pour être exploitable, que ce soit chez le blé (Branlard, 1980; Seguin, 1990) ou le colza (Lefort-Buson, 1986). Les hypothèses de fécondation aléatoire et d'absence de sélection nécessaires au calcul des coefficients de parenté de Malécot, hypothèses qui pèsent encore plus lourdement au niveau des autogames que des allogames, expliquent sans doute en partie ce résultat négatif. Une autre source d'erreur tient au manque de fiabilité des généalogies déclarées : une étude portant sur un échantillon important de blés français a en effet montré que $32 \%$ des diagrammes gluténines des lignées étudiées n'étaient pas compatibles avec les diagrammes des lignées ancêtres (Picard, 1990).

\section{Prédiction de l'hétérosis}

En dehors des distances biochimiques et généalogiques utilisées dans notre étude, des distances basées sur la variabilité des caractères agromorphologiques ont également été employées pour tenter de prédire l'hétérosis. Les résultats obtenus sont différents selon les auteurs. Chez le blé, Shamsuddin (1985), après avoir déterminé des groupes de lignées sur la base d'une distance agromorphologique, a montré que l'hétérosis était plus important lorsque les parents étaient choisis dans 2 groupes différents. Arunachalam et al (1984) obtiennent un résultat similaire pour l'arachide, avec cependant des hétérosis plus faibles lorsque les pa- 
rents sont choisis dans 2 groupes trop divergents. D'autres résultats bibliographiques indiquent une absence de liaison entre distances agromorphologiques et hétérosis, chez le pois chiche (Imitaz Ahmed Khan et al, 1991) et chez le colza (Lefort-Buson, 1986). Chez le mais, Smith et Smith (1989b) trouvent une corrélation significative entre une distance agromorphologique et l'hétérosis, mais le coefficient de détermination reste peu élevé. De toutes façons, l'utilisation de telles distances agromorphologiques est délicate (Smith et Smith, 1989a), car si les caractères qu'elles mettent en jeu sont généralement à déterminisme polygénique (ce qui permet d'obtenir une meilleure couverture du génome que dans le cas des distances biochimiques), l'expression de ces caractères est également souvent fortement influencée par le milieu.

Pour la prédiction de l'hétérosis, ce sont donc maintenant les distances basées sur les marqueurs moléculaires qui apparaissent comme les outils les plus prometteurs (Burr et al, 1983). Ces marqueurs moléculaires ont l'avantage de couvrir une grande proportion du génome et de ne pas être influencés par le milieu. Cependant, chez le maïs, plusieurs études récentes indiquent une absence de liaison entre des distances basées sur le polymorphisme des longueurs des fragments de restriction de I'ADN (RFLP) et l'hétérosis (Godshalk et al, 1990; Melchinger et al, 1990a,b), et les liaisons positives mises en évidence par Lee et al (1989) ou Smith et al (1990) pourraient être dues essentiellement au fait que le matériel qu'ils ont étudié incluait des hybrides entre lignées apparentées (combinaisons intragroupes hétérotiques). En fait, dans la mesure où les locus impliqués dans un caractère quantitatif sont localisés seulement dans quelques régions chromosomiques, le marquage aléatoire du génome par les marqueurs moléculaires n'est pas forcément approprié pour estimer la complémentarité à ces locus (Melchinger et al, 1990a,b), et il serait sans doute plus efficace de se focaliser sur un marquage spécifique des régions contribuant à l'hétérosis.

\section{CONCLUSION}

L'objectif de cette étude était de vérifier l'existence d'une relation entre les distances génétiques interparentales et l'hétérosis sur le rendement des hybrides correspondants, ceci en vue d'une meilleure connaissance de l'hétérosis. Or, le calcul de distances et d'apparentement s'est avéré inexploitable pour la prédiction de l'hétérosis sur le rendement.

Pour identifier les gènes, vraisemblablement nombreux, impliqués dans la vigueur hybride, une des voies possibles réside dans la création de lignées de substitution sur les différents chromosomes de chacun des trois génomes. Ces lignées seraient ensuite utilisées pour la création d'hybrides avec un parent receveur. Les combinaisons offrant un hétérosis élevé constitueraient un matériel de choix pour connaître les chromosomes et les séquences impliqués dans la supériorité phénotypique. Cependant, les conclusions sur la vigueur hybride risquent d'être liées à l'originalité des blés pris comme donneur et comme receveur.

\section{RÉFÉRENCES}

Arunachalam V, Bandyopadhyay A, Nigam SN, Gibbons RW (1984) Heterosis in relation to genetic divergence and specific combining ability in groundrut (Arachis Hypogaea L). Euphytica 33, 33-39

Branlard G (1980) Contribution à l'étude biochimique et génétique du polymorphisme des prolamines chez les Triticinae. Application à l'amélioration de Triticum aestivum ssp vulgare. Thèse de docteuringénieur de l'université de Clermont II, 89-92

Branlard G (1983) Study of genetic determination of 20 gliadin bands. Theor App/ Genet 64, 155-162

Brunel D (1985) Les distances génétiques : estimations et applications. III. Distances génétiques et hétérosis : utilisation des marqueurs moléculaires. In: Les distances génétiques, INRA, ed, 159-168

Burr B, Évola SC, Burr FA, Beckman JS (1983) The application of restriction fragment length polymorphisms to plant breeding. In: Genetic engineering: principles and methods (JK Setlow, A Hollaender, eds) Plenum Press, London, 5, 45-59

Charcosset $A$ (1990) Étude de l'hétérosis chez le maïs (Zea mays $L$ ) : prédiction de la valeur d'hybrides $F_{1}$ sur la base d'informations agronomiques, biochimiques et moléculaires. Thèse de docteur de l'Institut National Agronomique Paris-Grignon, $87 p+$ annexes

Charcosset A, Lefort-Buson M, Gallais A (1990) Use of top-cross designs for predicting performance of maize single cross hybrids. Maydica 35, 23-27

Cox TS, Murphy JP (1990) The effect of parental divergence on $F_{2}$ heterosis in winter wheat crosses. Theor Appl Genet 79, 241-250

Damerval C, Hébert $Y$, Vienne De D (1987) Is the polymorphism of protein amounts related to phenotypic variability. A comparison of two-dimensional 
electrophoresis data with morphological traits in maize. Theor Appl Genet 74, 194-202

East EM (1936) Heterosis. Genetics 21, 375-397

Ehiobu NG, Goddard ME, Taylor JF (1990) Prediction of heterosis in crosses between inbred lines of Drosophila melanogaster. Theor Appl Genet 80, 321325

Freeman GF (1919) Heredity of quantitative characters in wheat. Genetics 4, 1-93

Frei OM, Stuber CW, Goodman MM (1986) Uses of allozymes as genetic markers for predicting performance in maize single-cross hybrids. Crop Sci 26 , $37-42$

Fusakawa $H$ (1955) Studies on restoration and substitution of nucleus of Aegilotricum. Cytologica 20, 211-217

Godshalk EB, Lee M, Lamkey KR (1990) Relationship of restriction fragment length polymorphisms to single-cross hybrid performance of maize. 'Theor Appl Genet 80, 273-280

Heidrich-Sobrinho E, Cordeiro AR (1975) Codominant isoenzymic alleles as markers of genetic diversity correlated with heterosis in maize (Zea mays $\mathrm{L}$ ). Theor App/ Genet 46, 197-199

Imitiaz Ahmed Khan, Seema Imitiaz, Bashir Ahmed Malik (1991) Selection of diverse parents of chickpea (Cicer arietium $\mathrm{L}$ ) by multivariate analysis and the degree of heterosis of their $F_{1}$ hybrids. Euphytica 51, 227-233

Kihara (1951) Substitution of nucleus and its effects in genome manifestation. Cytologie 16, 177-193

Laemmli UK (1970) Cleavage of structural proteins during the assembly of the head of bacteriophage T4. Nature 227, 680-685

Lee $M$, Godshalk EB, Lamkey KR, Woodman WW (1989) Association of restriction fragment length polymorphisms among maize inbreds with agronomic performance of their crosses. Crop Sci 29 , 1067-1071

Lefort-Buson M (1985a) Mesures de la divergence génétique. I. Identité : parenté et consanguinité. In: Les distances génétiques, INRA ed, 13-21

Lefort-Buson $M$ (1985b) Distances génétiques et hétérosis. I. Mise en évidence d'une relation entre hétérosis et divergence génétique. In: Les distances génétiques, INRA ed, Paris, 111-118

Lefort-Buson M (1986) Hétérosis chez le colza oléagineux (Brassica napus $L$ ) : analyse génétique et prédiction. Thèse d'état de l'université Paris-Sud Orsay, $228 p$

Léonardi A, Damerval C, Hébert Y, Gallais A, Vienne, Vienne De D (1991) Association of protein amount polymorphism (PAP) among maize lines with performances of their hybrids. Theor Appl Genet 82, 552560

Malécot G (1948) Les mathématiques de l'hérédité. Masson et Cie, Paris
Mécham OK, Kasarda DD, Qualset CO (1978) Genetic aspects of wheat gliadin proteins. Biochem Genet 16, 831-853

Melchinger $A E$, Lee $M$, Lamkey $K R$, Hallauer $A R$, Woodman WL (1990a) Genetic diversity for restriction fragment length polymorphisms and heterosis for two diallel sets of maize inbreds. Theor Appl Genet 80, 488-496

Melchinger AE, Lee M, Lamkey KR, Woodman WL (1990b) Genetic diversity for restriction fragment length polymorphisms: relation to estimated genetic effects in maize inbreds. Crop Sci 30, 10331040

Moll RH, Salhuana WS, Robinson HF (1962) Heterosis and genetic diversity in variety crosses of maize. Crop Sci 2, 197-198

Moll RH, Lonnquist JH, Fortuna JV, Johnson CE (1965) The relationship of heterosis and genetic divergence in maize. Genetics 52, 139-144

Oury FX, Brabant $P$, Pluchard $P$, Bérard $P$, Rousset M (1990) Étude multilocale de blés hybrides : niveaux d'hétérosis et élaboration du rendement. Agronomie 10, 735-748

Paterniani E, Lonnquist JH (1963) Heterosis in interracial crosses of corn (Zea mays L). Crop Sci 3, 504507

Payne PI, Corfield KG, Blackman JA (1979) Identification of a high molecular weight subunits of glutenin whose presence correlates with bread making quality in wheats of related pedigree. Theor Appl Genet 55, 153-159

Payne PI, Holt LM, Jackson EA (1984) Genetical analysis of wheat endosperm storage proteins. In: Proc 2nd Int Workshop Gluten Proteins (Graveland A, Moonen JHE, eds) Wageningen, Pays-Bas, 111 119

Picard B (1990) Étude de la diversité biochimique et génétique du blé tendre : application à la connaissance de l'hétérosis. Thèse Univ Clermont II, $121 \mathrm{p}$ + annexes

Picard B, Branlard G, Oury FX, Rousset M (1992) Étude de la diversité biochimique et génétique du blé tendre. I. Comparaison de distances. Agronomie 12, 611-622

Séguin M (1990) Étude de l'hétérozygotie chez le blé tendre par l'électrophorèse bidimensionnelle des protéines totales et relation avec des caractères agronomiques. Thèse de I'Université Paris-Sud Orsay, $211 p$

Shamsuddin AKM (1985) Genetic diversity in relation to heterosis and combining ability in spring wheat. Theor Appl Genet 70, 306-308

Smith JSC, Smith OS (1989a) The description and assessment of distance between inbred lines of maize: I. The use of morphological traits as descriptors. Maydica 34, 141-150

Smith HSC, Smith OS (1989b) The description and assessment of distance between inbred lines of 
maize. II. The utility of morphological, biochemical and genetic descriptors and a scheme for the testing of distinctiveness between inbred lines. Maydica 34, 151-161

Smith OS, Smith JSC, Bowen SL, Tenborg RA, Wall SJ (1990) Similarities among a group of elite maize inbreds as measured by pedigree, $F_{1}$ grain yield, grain yield heterosis and RFLPs. Theor Appl Genet $80,833-840$
Sozinov AA, Poperelya FA (1980) Genetic classification of prolamines and its use for plant breeding. Ann Technol Agric 29, 229-245

Strauss SH (1986) Heterosis at allozyme locus under inbreeding and crossbreeding in Pinus attenuata. Genetics 113, 114-134

Xu NY, Wang XL (1982) A preliminary study on heterosis in wheat and isozyme analysis. Hubei Agric Sci $12,4-8$ 